\title{
Machine vision tool for real-time detection of defects on textile raw fabrics
}

\author{
Rocco Furferi, Lapo Governi
}

\begin{abstract}
This work describes an automated artificial vision inspection (AVI) system for real-time detection and classification of defects on textile raw fabrics. The tool (software + hardware) is directly attached to an appositely developed appraisal equipment machine (weave room monitoring system) and the inspection is performed online. The developed tool performs (1) the image acquisition of the raw fabric, (2) the extraction of some critical parameters from the acquired images, (3) an artificial neural network (ANN)-based approach able to detect and classify the most frequently occurring types of defects occurring on the raw fabric and (4) a standard image processing algorithm that allows the measurement of the geometric properties of the detected defects.

The reliability of the tool is about $90 \%$ (defect detected vs. effectively existing defects), that is, similar to the performance obtained by human experts. Once detected the defects are correctly classified in $88 \%$ of cases and their geometrical properties are measured with a sub-pixel precision.
\end{abstract}

